\title{
Myelitis in Systemic Lupus Erythematosus
}

Xiang-Yang $\mathrm{Li}^{1}$, Hai-Bing Xiao ${ }^{2}$, Pearl Pai ${ }^{1,3}$

1 Department of Nephrology, The University of Hong Kong-Shenzhen Hospital, Shenzhen, China.

2 Department of Neurology, The University of Hong Kong-Shenzhen Hospital, Shenzhen, China.

3 Department of Medicine, The University of Hong Kong - Queen Mary Hospital, Pokfulam Road, Hong Kong, China.

Correspondence: Pearl Pai, Department of Nephrology, University of Hong Kong-Shenzhen hospital, Shenzhen, Guangdong, China. 518053. E-mail: ppai1@hku.hk

\begin{abstract}
SLE-associated acute transverse myelitis (ATM) is a rare, but potentially severe complication of Systemic lupus erythematosus (SLE), and may lead to significant motor, sensory and autonomic dysfunctions in the central nervous system resulting in marked neurological deficits. It is important to recognize its clinical feature to allow timely diagnosis and management of this condition. In this review, we aimed to provide the reader with the understanding of its clinical presentation and classification, the underlying pathological, MRI (magnetic resonance imaging) appearance, and current status of management, with an emphasis on recent discoveries and advancements.
\end{abstract}

\section{Key Words}

transverse myelitis; myelopathy; systemic lupus erythematosus; pathology; magnetic resonance imaging; diagnosis; therapy; prognosis

\section{Introduction}

Systemic lupus erythematosus (SLE) may affect the nervous system at multiple levels. Neuropsychiatric systemic lupus erythematosus (NPSLE) encompasses a spectrum of symptoms and disorders that involve the central or peripheral nervous system during the disease process of SLE. In 1999, the American College of Rheumatology (ACR) developed a nomenclature system containing 19 NPSLE syndromes to facilitate recognition, diagnosis and classification, amongst which "myelopathy" is used to specify injury of the spinal cord[1]. The term "myelopathy" 
generally describes pathologies such as ischemia, compression, metabolic and inflammatory causes. When the spinal lesion is initiated and caused by inflammation, it is often termed "myelitis", but the two expressions are considered interchangeable in published literatures. The clinical presentations of myelitis comprise motor, sensory and autonomic dysfunctions. The severity varies according to the extent of spinal lesion and may range from mild extremity numbness, dysesthesia total sensory loss, weakness, paraplegia, and bowel and anal sphincter dysfunction. The term "acute transverse myelitis (ATM)" was first used in 1948 to describe the development of paraparesis with a thoracic sensory level in a patient with pneumonia and postinfectious myelitis[2]. In fact, the word "transverse" bears no relationship to the radiological or pathological lesion, but was solely used to highlight the importance of a spinal sensory level in reaching the diagnosis[3]. By 2002, the International Transverse Myelitis Consortium Working Group produced a set of diagnostic criteria and nosology for ATM and a new classification was proposed and adopted[4]. Based on this classification, ATM is broadly categorized as secondary or idiopathic. The latter can only be established when other disease-associated myelitis has been excluded. When the myelitis is caused by SLE[4], the condition is referred to SLE-associated ATM. This review examined the clinical features, pathological changes, differential diagnosis and therapy in SLE-associated ATM.

\section{Incidence and Prevalence}

The overall incidence of transverse myelitis is reportedly between 1 (severe) to 8 (mild) cases per million per year[3], with a bimodal peak between the ages of 10 to 19 years and 30 to 39 years; no gender or familial predisposition has been reported. The reported prevalence of SLE in the population is 200 to 1500 cases per million[5-7]. In Asia, Feng[8] has estimated the prevalence of SLE to be 500-1000 cases per million population. The incidence of SLE is also variable, ranging from 10 to 250 per million per year in North America, South America, Europe and Asia[5, 9, 10]. Myelitis is a relatively rare complication of SLE[11]. The incidence of ATM among SLE population was mainly based on case serials and was reportedly $1-2 \%[12,13]$, documented by literatures prior to 2000 ; and $0.9 \%[14]$ to $1.1 \%[15]$ according to more recent publications.

\section{Clinical manifestations and subtypes}

TM is characterized by lesions of inflammation within the spinal cord. Its clinical manifestations 
arise from resultant interruptions of motor, sensory, and autonomic pathways within and passing through the inflamed area. SLE is a systemic inflammatory disorder caused by autoimmune reactions, the pathomechanism of neurological damage of SLE- associated ATM is believed to share same features of its underlying disease[4]. The neurological deficit and clinical symptoms of SLE-associated ATM are not dissimilar to TM of other etiologies. These symptoms include acute or sub-acute paraparesis of the lower extremities, with initial flaccidity followed by spasticity. Most patients have a sensory level. Typical sensory dysfunctions include pain, dysesthesia, and paresthesia. Autonomic symptoms include increased urinary urgency, bladder and bowel incontinence, inability to void, incomplete evacuation with constipation, and sexual dysfunction[16]. The signs and symptoms depend upon the level of spine being involved. ATM can be further divided into two subgroups[17], an acute complete transverse myelitis (ACTM) or acute partial transverse myelitis (APTM). ACTM indicates moderate or severe symmetrical weakness and autonomic dysfunction attributable to a spinal level. APTM describes mild sensory and or motor dysfunction, bilateral or unilateral; when severe deficits are present, marked asymmetry is observed.

ATM may also be categorized as grey matter or white matter myelitis[18]. In the case of grey matter myelitis, conditions may deteriorate rapidly, reaching a clinical nadir within 6 hour with no subsequent improvement while white matter myelitis is characterized by the development of upper-motor neuron spasticity and hyperreflexia at the onset but the symptoms were less severe with slower progression.

\section{Pathological changes}

The pathological hallmark of TM is the presence of local collections of lymphocytes and monocytes, with varying degrees of demyelination, axonal injury, and astroglial and microglial activation within the spinal cord[19]. The pathogenesis of specific disease-associated TM is related to its primary disease[16]. Thus far, our understanding regarding the pathomechanism of NPSLE is limited to the injuries resulting from vasculopathy[20], auto-antibodies[21], cytokines and chemokines, and oxidative stress, nitric oxide, and interference with neurotransmission[22-25]. In 1975, a review of autopsy studies regarding the pathological changes in SLE-associated TM revealed various vascular changes within the spinal cord in 11 out of 12 cases. The abnormalities 
included ischemic necrosis, infarction or malacia in 8 cases, vasculitis without necrotic foci in 2 cases and degenerative lesions within the white matter with adventitia thickening in small arteries in 1 case. Vascular pathologies included perivascular lymphocytic infiltrations, proliferation of connective tissue, thrombi in small arteries and arterioles, and micro-extravasations within the spinal cord parenchyma[26]. The authors suggested that an autoimmunologic process was responsible for the vascular lesions in lupus myelitis. Other researchers demonstrated the presence of thrombosis, fibrinoid arteries, perivasculitis, spinal cord softening and peripheral white-matter degenerations at multiple spinal cord levels[27]. A more recent case report [2014] described the presence of intimal hyperplasia and obliteration of the small arteries and vasculitis (mononuclear cell infiltration and disruption of internal elastic lamina) in the affected case[28]. There are suggestions that the pathological damage in lupus-associated myelitis might be less prominent after intensive immunosuppressive therapy[29].

\section{Magnetic Resonance Imaging}

MRI is the modality of choice for the investigation of intramedullary lesions of the spinal cord. Both spinal and brain MRI are crucial in excluding spinal cord compression and in differentiating subtypes of acute demyelinating myelitis and other NPSLE disorders.

The detection of inflammatory lesion in the spinal cord can be facilitated by administering intravenous gadolinium on a T1-weighted image. However, the typical MRI appearance of ATM can be seen as a high intensity lesion detectable on a T2-weighted image, indicative of interstitial inflammation.

Under MRI, the affected spinal segments are described as "short" or "transverse" if less than 2-3 vertebral body segments are affected whereas "long" or "extensive" lesions involve more than 3 vertebral bodies. Both multiple sclerosis (MS) and neuromyelitis optica (NMO) may resemble ATM in its presentation. According to Weinshenker BG and co-workers[30], the involvement of more than three vertebral bodies was more common in NMO and helped to differentiate NMO from MS. In 2011, a panel committee of American Academy of Neurology endorsed the classification and recommended >3 vertebral bodies as a standard for the "longer" subtype of TM. Our group has reviewed the published literature regarding the length of spinal cord lesion of SLE-related ATM and MR imaging[31]. Among 63 cases of lupus-associated myelitis with MRI information, $71.4 \%$ of patients (45/63) had confirmed longitudinal lesion (more than three vertebral segments) whereas $28.6 \%(18 / 63)$ had transverse lesion (less or equal to three vertebral segments)[31]. Hence, it 
would appear that the spinal cord injury of SLE-associated ATM is more often longitudinal or extensive.

\section{Laboratory testings}

Besides MRI, the study of cerebral spinal fluid (CSF) is an established tool for detecting spinal cord inflammation. CSF pleocytosis and an increased Immunoglobulin $\mathrm{G}(\mathrm{IgG})$ index represent a classic inflammatory marker. Hence, both MRI and CSF studies may be used to ascertain inflammatory myelitis. Serological markers indicative of SLE include antinuclear antibodies (ANA), antidouble-strand antibody (anti-dsDNA), antibodies to extractable nuclear antigen (ENA), anti-Smith antibody (anti-Sm), and antiphospholipid antibodies (aPLs).

It was reported that $40-50 \%$ of NPSLE occurred in the presence of generalized disease activity of SLE[32]. It is important to look for activity of SLE by measuring full blood count, urinalysis, Coomb's test, complement profiles of $\mathrm{C} 3, \mathrm{C} 4, \mathrm{CH} 50$ and auto-antibodies. However, it was observed that SLE-ATM could occur in the absence of other disease activity[13, 33]. In a case series of 370 SLE patients, myelitis was associated with a lower SLE Disease Activity Score and European Consensus Lupus Activity Measurement (ECLAM) contrary to other CNS involvement with higher activity score[15].

In our previous study[31], out of 94 patients with SLE-associated ATM and reported disease activities, 61 myelitis (64.8\%) occurred during active disease (SLEDI>4, or SLAM>1) but 33 (35.1\%) occurred during low lupus activity (SLEDI $\leq 4$, or SLAM $\leq 1)$. It has been reported that SLE patients with ATM are more likely to possess aPLs. Moreover, aPL-induced thrombosis has been proposed as a major pathomechanism in the development of transverse myelitis in SLE[11, 34]. According to a large prospective study, all major aPLs (anticardiolipin antibodies, lupus anticoagulant, and B2-glucoprotein-1 inhibitor) and their titers should be measured to evaluate any thrombotic risk[35]. The findings of oligoclonal bands in the CSF and serum anti-aquaporin-4 immunoglobulin G (AQP4-IgG) may be used to differentiate SLE-associated ATM from MS and idiopathic NMO respectively.

\section{Diagnosis and Differential diagnosis}


The diagnosis of SLE-associated ATM is usually straightforward when the myelitis occurred in the background of SLE, while other causes have been excluded. Other secondary causes include CNS infection (e.g. herpes virus or mycoplasma), other autoimmune disease (e.g. ankylosing spondylitis, aPL syndrome, Behçet disease, mixed connective tissue disease, rheumatoid arthritis, scleroderma, Sjögren syndrome), as part of a paraneoplastic syndrome, neurosarcoidosis, or other multifocal neurological disease (e.g. MS and NMO).

It is noteworthy that the development of myelitis may be first manifestation of SLE. There has been reports that ATM was the first sign of underlying SLE in 7 out of 20 cases[36] and 5 out of 14 cases[13] of SLE subjects. In our previous report of 58 SLE myelitis cases, acute myelitis was the initial complaint in 12 of them (21\%)[31]. In these cases, it is essential to make a diagnosis of the underlying SLE process in order to manage the condition fully. But the heterogeneity of clinical manifestations poses a diagnostic challenge. In a case series of 20 patients, SLE was not diagnosed for 4 to 10.4 years after an ATM attack[36]. Hence, the immunological screening for SLE (e.g ANA, anti-dsDNA, anti-Sm, aPLs) is necessary in these cases.

We recommend using the Systemic Lupus International Collaborating Clinics (SLICC) classification criteria[37] or 1997 ACR (American Rheumatism Association) criteria[38] for the diagnosis of SLE. The two classification criteria complement each other in terms of diagnosing SLE accurately[39]. The differentiation of SLE-ATM from MS and NMO is beyond the scope of this article. MS and NMO are once considered separate entities from SLE-associated myelitis[4]. It is now observed that the both MS[40] and NMO[41] may coexist with SLE. It is further suggested that SLE and other systemic rheumatologic diseases may facilitate the pathogenesis of NMO spectrum disease (NMOSD) by allowing AQP4-IgG to access the CNS through a disrupted blood-brain barrier[41]. An international expert panel concluded that when a patient has clinical manifestations of ATM in the presence of serological and clinical features of both SLE and NMOSD (with AQP4-IgG positivity), it should be diagnosed as co-association of SLE and NMOSD, rather than a direct complication of SLE (SLE-related ATM)[42]. The MRI appearance of long and short TM may assist the differentiations of underlying cause $[43,44]$. This differentiation is important in guiding the appropriate therapy (see below).

\section{Therapy and management}

Thus far, the management of SLE-associated ATM has been mainly translated from treatment of 
SLE and that of idiopathic inflammatory myelitis. During the acute phase, treatment is aimed to suppress the inflammatory process and stop any disease progression. Longer-term goal is aimed to prevent cord damage or loss of function.

Intravenous pulse methylprednisolone ( 0.5 to $1 \mathrm{~g} /$ day for 3 days) and cyclophosphamide (800 to $1200 \mathrm{mg} / \mathrm{m}^{2}$ administered as a single pulse dose) are commonly used in the first line management of SLE[45] with major organ involvement and in idiopathic ATM[46]. There is evidence that the additional cyclophosphamide improves the efficacy[47], and is therefore recommended by the EULAR task force[48]. Plasma exchange (PE) is sometimes used in the treatment of severe auto-immune disease by removing autoantibodies, activated complement components, coagulation factors and cytokines. Positive benefits of PE have been reported in patients with NPSLE refractory to other treatments[49], although its efficacy in this condition needs to be further tested in randomized controlled trials. Empirically, glucocorticoids have been used in combination with other immunosuppressive agents that include mycophenolate, azathioprine, intravenous immunoglobulin (IVIG), and rituximab, in the management of SLE-ATM[13, 31, 36, 50, 51]. Interestingly, rituximab, a B cell-depleting monoclonal anti-CD20 antibody, has been reported to be effective in the treatment of new onset SLE-associated ATM[52] and in NPSLE refractory to standard immunosuppressive agents, plasma exchange, and/or immunoadsorption therapy[53]. The rituximab is typically administered in combination with glucocorticoid. In the setting of co-association of SLE and NMOSD, rituximab or azathioprine may be used as first line agent in an attempt to lower serum AQP4-IgG[54].

After the induction therapy with high dose intravenous glucocorticoid and cyclophosphamide, most patients are maintained on azathioprine, methotrexate, mycophenolate, or oral cyclophosphamide with or without low dose steroid[16]. Maintenance immunosuppressive treatment is usually recommended for 3 or more years although the optimal duration of maintenance therapy is yet to be defined. In cases of SLE with NMO, at least 5 years maintenance therapy is recommended[54].

The use of anticoagulation in SLE-associated ATM will need to be considered case by case. APL is thought to play a role in SLE-related ATM as evidenced by several pathological studies[25, 26, 28] and a large prospective study[35]. The use of anti-platelet and/or anticoagulation therapy is recommended for NPSLE related to antiphospholipid antibodies, and also for the management of 
ischemic optic neuropathy and refractory myelopathy[48]. The use of anticoagulant may be superior to anti-platelet therapy for secondary prevention of arterial event, as suggested by the EULAR task force[48]. However, in a review of 70 cases of SLE-associated ATM, Katsiari et al reported that they were unable to demonstrate any benefit of anticoagulation in SLE-associated ATM[55]. This study was limited by the relatively small sample size, retrospective design, and that the three subtypes of aPLs and their titers were not measured concurrently. It has been suggested that the presence of more subtypes and a higher titer of aPL may predispose to more thrombotic damage[35], thus making the use of anticoagulant more favorable. However, both subdural hematoma and spinal cord bleeding have been reported in lupus-related myelopathy[27], it is therefore important to weigh any pros and cons regarding anticoagulation therapy[48]. We may consider a target INR of 2.0-3.0 (moderate) in lupus ATM complicated with APS; but where there is a high risk of arterial thrombosis or recurrent thrombosis[56], immobility or hyper-coagulation states, to consider a higher target INR of 3.1-4.0.

\section{Prognosis}

Patients with SLE have an almost 5-fold increased risk of death compared with the general population. And involvement with major organ(s) denotes a worse prognosis[56]. Up to now, limited retrospective studies have been published concerning the outcomes and prognosis of SLE-related ATM. The prognosis of SLE-associated ATM is unfavorable(tetraplegia, or paraplegia, paraparesis, sensory loss, sphincter dysfunction) in up to $25 \%-66.7 \%[13,36,51]$ with various definition of outcome and observation period. Furthermore, there is a high rate of recurrence, reported between 21\%-55\%[57] and 50\%[36]. Partial remission has been reported between $50 \%-62.5 \%[13,33,36]$ and complete recovery has been documented as $7.1 \%-27.8 \%[13,36]$ with diverse observation period. There is a link between the severity of the motor symptom at the onset and prognosis; those with more severe symptoms such as paraplegia, predicts a poorer prognosis $[18,36,50]$. It was advised that the earlier administration of effective anti-inflammatory therapy (e.g. glucocorticoids) was associated with better neurological outcome[50].

Like SLE itself, SLE-related ATM is a heterogeneous entity, hence well-written case report may provide a useful source of information. Three cases of gestational lupus ATM were reported in the literature at week 22[58], week 16[59] and week 13[60] of the pregnancy, respectively. All three women were in their second pregnancy. They were treated with oral prednisone or 
prednisolone[59,60], pulse methylprednisolone[58], azathioprine[60] and plasma exchange therapy[60]. All three women underwent cesarean section to deliver three healthy female neonates but only one was delivered at full-term[58]; the other two were premature births. One woman developed permanent neurological deficit and required assistance for daily activities after delivery[58], while the other two women recovered well. In another case report, Nasir and colleagues[61] described a 48-year-old woman who experienced 19 attacks of myelitis during her 13-year history of SLE; all attacks had occurred without any other signs of lupus activity. She responded well to pulse methylprednisolone earlier on but less so in her later attacks.

Unfortunately, she became paraplegic after her last attack; a trial of rituximab had no benefit.

\section{Conclusion}

ATM is a relative rare association of SLE. The manifestation of SLE-associated ATM resembles that of idiopathic acute myelitis. Its pathological changes include vasculitis, perivasculitis, thrombosis, ischemic necrosis, infarction, degeneration etc. SLE-related ATM more often manifests as longitudinal myelitis on MRI and it may occur in low SLE disease activity. The treatment includes high dose glucocorticoids and intravenous cyclophosphamide, PE, IVIG and concomitant other immunosuppressive agents. Overall, the prognosis is not favorable, and recurrent rate is high during the cause of disease.

Funding: This study was funded by Shenzhen Science, Technology and Innovation Committee

Conflict of Interest: Not declared. 


\section{Reference}

1. The American College of Rheumatology nomenclature and case definitions for neuropsychiatric lupus syndromes. Arthritis Rheum 1999, 42(4):599-608.

2. Suchett-Kaye Al: Acute transverse myelitis complicating pneumonia; report of a case. Lancet 1948, 2(6524):417.

3. Scott TF, Frohman EM, De Seze J, Gronseth GS, Weinshenker BG: Evidence-based guideline: clinical evaluation and treatment of transverse myelitis: report of the Therapeutics and Technology Assessment Subcommittee of the American Academy of Neurology. Neurology 2011, 77(24):2128-2134.

4. Proposed diagnostic criteria and nosology of acute transverse myelitis. Neurology 2002, 59(4):499-505.

5. Pons-Estel GJ, Alarcon GS, Scofield L, Reinlib L, Cooper GS: Understanding the epidemiology and progression of systemic lupus erythematosus. Semin Arthritis Rheum 2010, 39(4):257-268.

6. Lawrence RC, Helmick CG, Arnett FC, Deyo RA, Felson DT, Giannini EH, Heyse SP, Hirsch R, Hochberg MC, Hunder GG et al: Estimates of the prevalence of arthritis and selected musculoskeletal disorders in the United States. Arthritis Rheum 1998, 41(5):778-799.

7. Chakravarty EF, Bush TM, Manzi S, Clarke AE, Ward MM: Prevalence of adult systemic lupus erythematosus in California and Pennsylvania in 2000: estimates obtained using hospitalization data. Arthritis Rheum 2007, 56(6):2092-2094.

8. Feng PH: Systemic lupus erythematosus: the face of Asia. Ann N Y Acad Sci 2007, 1108:114-120.

9. Danchenko N, Satia JA, Anthony MS: Epidemiology of systemic lupus erythematosus: a comparison of worldwide disease burden. Lupus 2006, 15(5):308-318.

10. Peschken CA, Esdaile JM: Rheumatic diseases in North America's indigenous peoples. Semin Arthritis Rheum 1999, 28(6):368-391.

11. Sanna G, Bertolaccini ML, Cuadrado MJ, Laing H, Khamashta MA, Mathieu A, Hughes GR: Neuropsychiatric manifestations in systemic lupus erythematosus: prevalence and association with antiphospholipid antibodies. J Rheumatol 2003, 30(5):985-992.

12. West SG: Neuropsychiatric lupus. Rheum Dis Clin North Am 1994, 20(1):129-158.

13. Kovacs B, Lafferty TL, Brent LH, DeHoratius RJ: Transverse myelopathy in systemic lupus erythematosus: an analysis of 14 cases and review of the literature. Ann Rheum Dis 2000, 59(2):120-124.

14. Bortoluzzi A, Scire CA, Bombardieri S, Caniatti L, Conti F, De Vita S, Doria A, Ferraccioli G, Gremese E, Mansutti $E$ et al: Development and validation of a new algorithm for attribution of neuropsychiatric events in systemic lupus erythematosus. Rheumatology (Oxford) 2015, 54(5):891-898.

15. Kampylafka El, Alexopoulos H, Kosmidis ML, Panagiotakos DB, Vlachoyiannopoulos PG, Dalakas MC, Moutsopoulos HM, Tzioufas AG: Incidence and prevalence of major central nervous system involvement in systemic lupus erythematosus: a 3-year prospective study of 370 patients. PLoS One 2013, 8(2):e55843.

16. Kaplin Al, Krishnan C, Deshpande DM, Pardo CA, Kerr DA: Diagnosis and management of acute myelopathies. Neurologist 2005, 11(1):2-18.

17. Scott TF: Nosology of idiopathic transverse myelitis syndromes. Acta Neurol Scand 2007, 115(6):371-376.

18. Birnbaum J, Petri M, Thompson R, Izbudak I, Kerr D: Distinct subtypes of myelitis in systemic lupus erythematosus. Arthritis Rheum 2009, 60(11):3378-3387.

19. Wingerchuk DM, Lennon VA, Lucchinetti CF, Pittock SJ, Weinshenker BG: The spectrum of neuromyelitis 
optica. Lancet Neurol 2007, 6(9):805-815.

20. Hammad A, Tsukada Y, Torre N: Cerebral occlusive vasculopathy in systemic lupus erythematosus and speculation on the part played by complement. Ann Rheum Dis 1992, 51(4):550-552.

21. Colasanti T, Delunardo F, Margutti P, Vacirca D, Piro E, Siracusano A, Ortona E: Autoantibodies involved in neuropsychiatric manifestations associated with systemic lupus erythematosus. J Neuroimmunol 2009, 212(1-2):3-9.

22. Vincent FB, Northcott M, Hoi A, Mackay F, Morand EF: Association of serum B cell activating factor from the tumour necrosis factor family (BAFF) and a proliferation-inducing ligand (APRIL) with central nervous system and renal disease in systemic lupus erythematosus. Lupus 2013, 22(9):873-884.

23. George-Chandy A, Trysberg E, Eriksson K: Raised intrathecal levels of APRIL and BAFF in patients with systemic lupus erythematosus: relationship to neuropsychiatric symptoms. Arthritis Res Ther 2008, 10(4):R97.

24. Lu XY, Zhu CQ, Qian J, Chen XX, Ye S, Gu YY: Intrathecal cytokine and chemokine profiling in neuropsychiatric lupus or lupus complicated with central nervous system infection. Lupus 2010, 19(6):689-695.

25. Fragoso-Loyo H, Richaud-Patin Y, Orozco-Narvaez A, Davila-Maldonado L, Atisha-Fregoso Y, Llorente L, Sanchez-Guerrero J: Interleukin-6 and chemokines in the neuropsychiatric manifestations of systemic lupus erythematosus. Arthritis Rheum 2007, 56(4):1242-1250.

26. Andrianakos AA, Duffy J, Suzuki M, Sharp JT: Transverse myelopathy in systemic lupus erythematosus. Report of three cases and review of the literature. Ann Intern Med 1975, 83(5):616-624.

27. Provenzale J, Bouldin TW: Lupus-related myelopathy: report of three cases and review of the literature. $J$ Neurol Neurosurg Psychiatry 1992, 55(9):830-835.

28. Tono T, Nagai T, Hoshiyama T, Sakuma Y, Wada T, Tanaka S, Hirohata S: Transverse myelitis extended to disseminated encephalitis in systemic lupus erythematosus: Histological evidence for vasculitis. Mod Rheumatol 2014:1-5.

29. Lukjanowicz M, Brzosko M: Myelitis in the course of systemic lupus erythematosus: review. Pol Arch Med Wewn 2009, 119(1-2):67-72.

30. Weinshenker BG, Wingerchuk DM, Vukusic S, Linbo L, Pittock SJ, Lucchinetti CF, Lennon VA: Neuromyelitis optica IgG predicts relapse after longitudinally extensive transverse myelitis. Ann Neurol 2006, 59(3):566-569.

31. Li XY, Xiao P, Xiao HB, Zhang LJ, Pai P, Chu P, Chan TM: Myelitis in systemic lupus erythematosus frequently manifests as longitudinal and sometimes occurs at low disease activity. Lupus 2014, 23(11):1178-1186.

32. Pendleton A, Arden N, Dougados M, Doherty M, Bannwarth B, Bijlsma JW, Cluzeau F, Cooper C, Dieppe PA, Gunther KP et al: EULAR recommendations for the management of knee osteoarthritis: report of a task force of the Standing Committee for International Clinical Studies Including Therapeutic Trials (ESCISIT). Ann Rheum Dis 2000, 59(12):936-944.

33. Espinosa G, Mendizabal A, Minguez S, Ramo-Tello C, Capellades J, Olive A, Cervera R: Transverse myelitis affecting more than 4 spinal segments associated with systemic lupus erythematosus: clinical, immunological, and radiological characteristics of 22 patients. Semin Arthritis Rheum 2010, 39(4):246-256.

34. Lavalle C, Pizarro S, Drenkard C, Sanchez-Guerrero J, Alarcon-Segovia D: Transverse myelitis: a manifestation of systemic lupus erythematosus strongly associated with antiphospholipid antibodies. J Rheumatol 1990, 17(1):34-37.

35. Alarcon-Segovia D, Deleze M, Oria CV, Sanchez-Guerrero J, Gomez-Pacheco L, Cabiedes J, Fernandez L, Ponce de Leon S: Antiphospholipid antibodies and the antiphospholipid syndrome in systemic lupus erythematosus. A prospective analysis of 500 consecutive patients. Medicine (Baltimore) 1989, 68(6):353-365. 
36. Saison J, Costedoat-Chalumeau N, Maucort-Boulch D, Iwaz J, Marignier R, Cacoub P, Vital-Durand D, Hot A, Tebib J, Aumaitre 0 et al: Systemic lupus erythematosus-associated acute transverse myelitis: manifestations, treatments, outcomes, and prognostic factors in 20 patients. Lupus 2015, 24(1):74-81.

37. Petri M, Orbai AM, Alarcon GS, Gordon C, Merrill JT, Fortin PR, Bruce IN, Isenberg D, Wallace DJ, Nived 0 et al: Derivation and validation of the Systemic Lupus International Collaborating Clinics classification criteria for systemic lupus erythematosus. Arthritis Rheum 2012, 64(8):2677-2686.

38. Hochberg MC: Updating the American College of Rheumatology revised criteria for the classification of systemic lupus erythematosus. Arthritis Rheum 1997, 40(9):1725.

39. Pons-Estel GJ, Wojdyla D, McGwin G, Jr., Magder LS, Petri MA, Pons-Estel BA, Alarcon GS: The American College of Rheumatology and the Systemic Lupus International Collaborating Clinics classification criteria for systemic lupus erythematosus in two multiethnic cohorts: a commentary. Lupus 2014, 23(1):3-9.

40. Fanouriakis A, Mastorodemos V, Pamfil C, Papadaki E, Sidiropoulos P, Plaitakis A, Amoiridis G, Bertsias G, Boumpas DT: Coexistence of systemic lupus erythematosus and multiple sclerosis: prevalence, clinical characteristics, and natural history. Semin Arthritis Rheum 2014, 43(6):751-758.

41. Wingerchuk DM, Weinshenker BG: The emerging relationship between neuromyelitis optica and systemic rheumatologic autoimmune disease. Mult Scler 2012, 18(1):5-10.

42. Wingerchuk DM, Banwell B, Bennett JL, Cabre P, Carroll W, Chitnis T, de Seze J, Fujihara K, Greenberg B, Jacob A et al: International consensus diagnostic criteria for neuromyelitis optica spectrum disorders. Neurology 2015, 85(2):177-189.

43. Goh C, Desmond PM, Phal PM: MRI in transverse myelitis. J Magn Reson Imaging 2014, 40(6):1267-1279.

44. Kim HJ, Paul F, Lana-Peixoto MA, Tenembaum S, Asgari N, Palace J, Klawiter EC, Sato DK, de Seze J, Wuerfel J et al: MRI characteristics of neuromyelitis optica spectrum disorder: an international update. Neurology 2015, 84(11):1165-1173.

45. Parker BJ, Bruce IN: High dose methylprednisolone therapy for the treatment of severe systemic lupus erythematosus. Lupus 2007, 16(6):387-393.

46. Greenberg BM, Thomas KP, Krishnan C, Kaplin Al, Calabresi PA, Kerr DA: Idiopathic transverse myelitis: corticosteroids, plasma exchange, or cyclophosphamide. Neurology 2007, 68(19):1614-1617.

47. Barile-Fabris L, Ariza-Andraca R, Olguin-Ortega L, Jara LJ, Fraga-Mouret A, Miranda-Limon JM, Fuentes de la Mata J, Clark P, Vargas F, Alocer-Varela J: Controlled clinical trial of IV cyclophosphamide versus IV methylprednisolone in severe neurological manifestations in systemic lupus erythematosus. Ann Rheum Dis 2005, 64(4):620-625.

48. Bertsias GK, loannidis JP, Aringer M, Bollen E, Bombardieri S, Bruce IN, Cervera R, Dalakas M, Doria A, Hanly JG et al: EULAR recommendations for the management of systemic lupus erythematosus with neuropsychiatric manifestations: report of a task force of the EULAR standing committee for clinical affairs. Ann Rheum Dis 2010, 69(12):2074-2082.

49. Neuwelt CM: The role of plasmapheresis in the treatment of severe central nervous system neuropsychiatric systemic lupus erythematosus. Ther Apher Dial 2003, 7(2):173-182.

50. Lu X, Gu Y, Wang Y, Chen S, Ye S: Prognostic factors of lupus myelopathy. Lupus 2008, 17(4):323-328.

51. Tellez-Zenteno JF, Remes-Troche JM, Negrete-Pulido RO, Davila-Maldonado L: Longitudinal myelitis associated with systemic lupus erythematosus: clinical features and magnetic resonance imaging of six cases. Lupus 2001, 10(12):851-856.

52. Ye Y, Qian J, Gu Y, Chen X, Ye S: Rituximab in the treatment of severe lupus myelopathy. Clin Rheumatol 2011, 30(7):981-986.

53. Tokunaga M, Saito K, Kawabata D, Imura Y, Fujii T, Nakayamada S, Tsujimura S, Nawata M, Iwata S, Azuma T et al: Efficacy of rituximab (anti-CD20) for refractory systemic lupus erythematosus involving the central nervous system. Ann Rheum Dis 2007, 66(4):470-475. 
54. Trebst C, Jarius S, Berthele A, Paul F, Schippling S, Wildemann B, Borisow N, Kleiter I, Aktas O, Kumpfel T: Update on the diagnosis and treatment of neuromyelitis optica: recommendations of the Neuromyelitis Optica Study Group (NEMOS). J Neurol 2014, 261(1):1-16.

55. Katsiari CG, Giavri I, Mitsikostas DD, Yiannopoulou KG, Sfikakis PP: Acute transverse myelitis and antiphospholipid antibodies in lupus. No evidence for anticoagulation. Eur J Neurol 2011, 18(4):556-563.

56. Bertsias G, loannidis JP, Boletis J, Bombardieri S, Cervera R, Dostal C, Font J, Gilboe IM, Houssiau F, Huizinga $T$ et al: EULAR recommendations for the management of systemic lupus erythematosus. Report of a Task Force of the EULAR Standing Committee for International Clinical Studies Including Therapeutics. Ann Rheum Dis 2008, 67(2):195-205.

57. Schulz SW, Shenin M, Mehta A, Kebede A, Fluerant M, Derk CT: Initial presentation of acute transverse myelitis in systemic lupus erythematosus: demographics, diagnosis, management and comparison to idiopathic cases. Rheumatol Int 2012, 32(9):2623-2627.

58. Inslicht DV, Stein AB, Pomerantz F, Ragnarsson KT: Three women with lupus transverse myelitis: case reports and differential diagnosis. Arch Phys Med Rehabil 1998, 79(4):456-459.

59. Marabani M, Zoma A, Hadley D, Sturrock RD: Transverse myelitis occurring during pregnancy in a patient with systemic lupus erythematosus. Ann Rheum Dis 1989, 48(2):160-162.

60. Moranne O, Hachulla E, Valat AS, Sotoares G, Pagniez D, Boulanger E: Longitudinal myelitis in a pregnant patient with SLE. Am J Med 2004, 116(5):355-357.

61. Nasir S, Kerr DA, Birnbaum J: Nineteen episodes of recurrent myelitis in a woman with neuromyelitis optica and systemic lupus erythematosus. Arch Neurol 2009, 66(9):1160-1163. 